\title{
Correction to: Trends in use of and complications from intrauterine contraceptive devices and tubal ligation or occlusion
}

Brandon Howard ${ }^{1 *}$, ElizaBeth Grubb², Maureen J. Lage ${ }^{3}$ and Boxiong Tang ${ }^{4}$

As result of a query from a reader, the authors reviewed the data in Table 2 of the original article [1] and found a number of errors in the prior construction of this data table. A formatting error has also been noticed in Table 1.

The correct version of Table 1 and Table 2 is published in this erratum.

\footnotetext{
Author details

${ }^{1}$ Teva Global Medical Affairs, 41 Moores Road, Frazer, PA 19355, USA. ${ }^{2}$ Teva Global Health Economics \& Outcomes Research, 11100 Nall Ave, Overland Park, KS 66211, USA. ${ }^{3}$ HealthMetrics Outcomes Research, 27576 River Reach Dr., Bonita Springs, FL 34134, USA. ${ }^{4}$ Teva Global Health Economics \&

Outcomes Research, 41 Moores Road, Frazer, PA 19355, USA.
}

Received: 11 September 2017 Accepted: 19 September 2017

Published online: 06 October 2017

\section{Reference}

1. Howard, et al. Trends in use of and complications from intrauterine

contraceptive devices and tubal ligation or occlusion. Reprod Health. 2017;14:70 doi:10.1186/s12978-017-0334-1.

\footnotetext{
* Correspondence: Brandihoward@gmail.com

${ }^{1}$ Teva Global Medical Affairs, 41 Moores Road, Frazer, PA 19355, USA
} 
Table 1 Rate of IUD insertion and tubal sterilization over time

\begin{tabular}{|c|c|c|c|c|}
\hline & & Copper IUD & LNG IUD & Tubal Sterilization \\
\hline Year & Total N & n (\%) & n (\%) & n (\%) \\
\hline 2006 & $1,907,748$ & $3454(0.18)$ & $12,028(0.63)$ & $14,887(0.78)$ \\
\hline Age 15 to $19 y$ & 293,354 & $52(0.02)$ & $216(0.07)$ & $38(0.01)$ \\
\hline Age 20 to 24 y & 220,950 & $330(0.15)$ & $1257(0.57)$ & $451(0.20)$ \\
\hline Age 25 to $34 \mathrm{y}$ & 566,152 & $1735(0.31)$ & $6230(1.10)$ & $6642(1.17)$ \\
\hline Age 35 to $45 y$ & 827,292 & $1337(0.16)$ & $4325(0.52)$ & 7756 (0.94) \\
\hline 2007 & $1,940,301$ & $3803(0.20)$ & $16,789(0.87)$ & $14,769(0.76)$ \\
\hline Age 15 to $19 y$ & 299,599 & $79(0.03)$ & $416(0.14)$ & $42(0.01)$ \\
\hline Age 20 to $24 \mathrm{y}$ & 224,324 & $403(0.18)$ & $1813(0.81)$ & $444(0.20)$ \\
\hline Age 25 to $34 y$ & 583,955 & $1964(0.34)$ & $8533(1.46)$ & $6471(1.11)$ \\
\hline Age 35 to $45 y$ & 832,423 & $1357(0.16)$ & $6027(0.72)$ & $7812(0.94)$ \\
\hline 2008 & $2,001,739$ & $4474(0.22)$ & $24,276(1.21)$ & $14,667(0.73)$ \\
\hline Age 15 to $19 y$ & 308,311 & $102(0.03)$ & $690(0.22)$ & $21(0.01)$ \\
\hline Age 20 to $24 \mathrm{y}$ & 237,192 & $455(0.19)$ & $2845(1.2)$ & $410(0.17)$ \\
\hline Age 25 to $34 \mathrm{y}$ & 615,537 & $2368(0.38)$ & $12,545(2.04)$ & $6473(1.05)$ \\
\hline Age 35 to $45 y$ & 840,699 & 1549 (0.18) & 8196 (0.97) & 7763 (0.92) \\
\hline 2009 & $2,016,916$ & $4868(0.24)$ & $24,811(1.23)$ & $14,881(0.74)$ \\
\hline Age 15 to $19 y$ & 312,431 & $93(0.03)$ & $777(0.25)$ & $37(0.01)$ \\
\hline Age 20 to $24 \mathrm{y}$ & 237,723 & $460(0.19)$ & $2920(1.23)$ & $394(0.17)$ \\
\hline Age 25 to $34 \mathrm{y}$ & 625,323 & $2578(0.41)$ & $12,614(2.02)$ & $6232(1.00)$ \\
\hline Age 35 to $45 y$ & 841,439 & $1737(0.21)$ & $8500(1.01)$ & $8218(0.98)$ \\
\hline 2010 & $1,870,675$ & $5246(0.28)$ & $20,639(1.10)$ & $13,313(0.71)$ \\
\hline Age 15 to $19 y$ & 289,736 & $142(0.05)$ & $677(0.23)$ & $37(0.01)$ \\
\hline Age 20 to 24 y & 222,812 & $551(0.25)$ & $2101(0.94)$ & $243(0.11)$ \\
\hline Age 25 to $34 \mathrm{y}$ & 574,702 & $2782(0.48)$ & $10,316(1.8)$ & $5485(0.95)$ \\
\hline Age 35 to $45 y$ & 783,425 & $1771(0.23)$ & 7545 (0.96) & $7548(0.96)$ \\
\hline 2011 & $1,909,316$ & $4682(0.25)$ & $22,035(1.15)$ & $12,560(0.66)$ \\
\hline Age 15 to $19 y$ & 295,377 & $116(0.04)$ & $762(0.26)$ & $24(0.01)$ \\
\hline Age 20 to 24 y & 265,891 & $601(0.23)$ & $2648(1.00)$ & $280(0.11)$ \\
\hline Age 25 to $34 y$ & 575,729 & $2451(0.43)$ & $10,694(1.86)$ & $5102(0.89)$ \\
\hline Age 35 to $45 y$ & 772,319 & $1514(0.20)$ & 7931 (1.03) & 7154 (0.93) \\
\hline $\begin{array}{l}P \text { Value for Trend Over Time } \\
\text { in the Overall Population }\end{array}$ & & $<0.0001$ & $<0.0001$ & $<0.0001$ \\
\hline
\end{tabular}


Table 2 Complications and side effects associated with IUD insertion and tubal sterilization over time

\begin{tabular}{|c|c|c|c|}
\hline & Copper IUD & LNG IUD & Tubal Sterilization \\
\hline Complications/Side Effect & n (\%) & $\mathrm{n}(\%)$ & n (\%) \\
\hline \multicolumn{4}{|l|}{ Amenorrhea (ICD-9 626.0) } \\
\hline Total (2006-2011) & $2385(8.99)^{\mathrm{ab}}$ & $9077(7.53)^{\mathrm{ac}}$ & $9410(11.06)^{b c}$ \\
\hline 2006 & $308(8.92)$ & $941(7.82)$ & $1638(11.00)$ \\
\hline 2007 & $381(10.02)$ & $1278(7.61)$ & $1614(10.93)$ \\
\hline 2008 & $412(9.21)$ & $1873(7.72)$ & $1700(11.59)$ \\
\hline 2009 & $448(9.20)$ & $1845(7.44)$ & $1658(11.14)$ \\
\hline 2010 & $452(8.62)$ & $1518(7.36)$ & $1429(10.73)$ \\
\hline 2011 & $384(8.20)$ & $1622(7.36)$ & $1371(10.92)$ \\
\hline$P$ Value & 0.0775 & 0.4191 & 0.2768 \\
\hline \multicolumn{4}{|l|}{ Anemia (ICD-9 280.xx) } \\
\hline Total (2006-2011) & $595(2.24)^{b}$ & $2832(2.35)^{c}$ & $2823(3.32)^{b c}$ \\
\hline 2006 & $67(1.94)$ & $279(2.32)$ & $435(2.92)$ \\
\hline 2007 & $78(2.05)$ & $386(2.30)$ & $433(2.93)$ \\
\hline 2008 & $106(2.37)$ & $538(2.22)$ & $492(3.35)$ \\
\hline 2009 & $119(2.44)$ & $600(2.42)$ & $513(3.45)$ \\
\hline 2010 & $135(2.57)$ & $512(2.48)$ & $481(3.61)$ \\
\hline 2011 & $90(1.92)$ & $517(2.35)$ & 469 (3.73) \\
\hline$P$ Value & 0.1496 & 0.5243 & 0.0001 \\
\hline \multicolumn{4}{|l|}{ Dysmenorrhea (ICD-9 625.3) } \\
\hline Total (2006-2011) & $723(2.73)^{\mathrm{ab}}$ & $3909(3.24)^{a c}$ & $3678(4.32)^{b c}$ \\
\hline 2006 & $90(2.61)$ & $378(3.14)$ & $587(3.94)$ \\
\hline 2007 & $85(2.24)$ & $566(3.37)$ & $591(4.00)$ \\
\hline 2008 & $132(2.95)$ & $723(2.98)$ & $598(4.08)$ \\
\hline 2009 & $139(2.86)$ & $807(3.25)$ & $647(4.35)$ \\
\hline 2010 & $137(2.61)$ & $692(3.35)$ & $649(4.87)$ \\
\hline 2011 & $140(2.99)$ & $743(3.37)$ & $606(4.82)$ \\
\hline$P$ Value & 0.6388 & 0.1234 & $<0.0001$ \\
\hline \multicolumn{4}{|c|}{ Heavy Menstrual Bleeding (ICD-9 626.2) } \\
\hline Total (2006-2011) & $1370(5.16)^{\mathrm{ab}}$ & $10,204(8.46)^{a c}$ & $11,381(13.38)^{\mathrm{bc}}$ \\
\hline 2006 & $204(5.91)$ & $1048(8.71)$ & $1687(11.33)$ \\
\hline 2007 & $198(5.21)$ & $1406(8.37)$ & $1750(11.85)$ \\
\hline 2008 & $224(5.01)$ & $1846(7.60)$ & $1922(13.10)$ \\
\hline 2009 & $256(5.26)$ & $1992(8.03)$ & $2045(13.74)$ \\
\hline 2010 & $261(4.98)$ & $1881(9.11)$ & $2006(15.07)$ \\
\hline 2011 & $227(4.85)$ & $2031(9.22)$ & $1971(15.69)$ \\
\hline$P$ Value & 0.3527 & $<0.0001$ & $<0.0001$ \\
\hline \multicolumn{4}{|l|}{ Infection (ICD-9 998.5×) } \\
\hline Total (2006-2011) & $15(0.06)$ & $88(0.07)^{c}$ & $24(0.03)^{c}$ \\
\hline 2006 & $4(0.12)$ & $12(0.10)$ & $2(0.01)$ \\
\hline 2007 & $1(0.03)$ & $14(0.08)$ & $2(0.01)$ \\
\hline 2008 & $6(0.13)$ & $15(0.06)$ & $7(0.05)$ \\
\hline 2009 & $2(0.04)$ & $25(0.10)$ & $4(0.03)$ \\
\hline 2010 & $0(0.00)$ & $14(0.07)$ & $4(0.03)$ \\
\hline
\end{tabular}


Table 2 Complications and side effects associated with IUD insertion and tubal sterilization over time (Continued)

\begin{tabular}{|c|c|c|c|}
\hline & Copper IUD & LNG IUD & Tubal Sterilization \\
\hline Complications/Side Effect & n (\%) & n (\%) & n (\%) \\
\hline 2011 & $2(0.04)$ & $8(0.04)$ & $5(0.04)$ \\
\hline$P$ Value & 0.0543 & 0.1255 & 0.4301 \\
\hline \multicolumn{4}{|l|}{ Menorrhagia (ICD-9 627.0) } \\
\hline Total (2006-2011) & $53(0.20)^{a b}$ & $528(0.44)^{a c}$ & $640(0.75)^{\mathrm{bc}}$ \\
\hline 2006 & $12(0.35)$ & $48(0.40)$ & $88(0.59)$ \\
\hline 2007 & $6(0.16)$ & $58(0.35)$ & $94(0.64)$ \\
\hline 2008 & $11(0.25)$ & $84(0.35)$ & $92(0.63)$ \\
\hline 2009 & $12(0.25)$ & $89(0.36)$ & $119(0.80)$ \\
\hline 2010 & $8(0.15)$ & $121(0.59)$ & $121(0.91)$ \\
\hline 2011 & $4(0.09)$ & $128(0.58)$ & $126(1.00)$ \\
\hline$P$ Value & 0.1181 & $<0.0001$ & $<0.0001$ \\
\hline \multicolumn{4}{|l|}{ Ovarian Cyst (ICD-9 620.2) } \\
\hline Total (2006-2011) & $1157(4.36)^{\mathrm{ab}}$ & $6340(5.26)^{a c}$ & $6063(7.13)^{\mathrm{bc}}$ \\
\hline 2006 & $140(4.05)$ & $539(4.48)$ & $1045(7.02)$ \\
\hline 2007 & $155(4.08)$ & $786(4.68)$ & $990(6.70)$ \\
\hline 2008 & $211(4.72)$ & $1268(5.22)$ & $1076(7.34)$ \\
\hline 2009 & $209(4.29)$ & $1400(5.64)$ & 1039 (6.98) \\
\hline 2010 & $223(4.25)$ & $1142(5.53)$ & $1003(7.53)$ \\
\hline 2011 & $219(4.68)$ & $1205(5.47)$ & $910(7.25)$ \\
\hline$P$ Value & 0.5197 & $<0.0001$ & 0.0975 \\
\hline \multicolumn{4}{|c|}{ Pelvic Inflammatory Disease (ICD-9 614.xx-616.xx) } \\
\hline Total (2006-2011) & $93(0.35)$ & $334(0.28)^{c}$ & $319(0.37)^{c}$ \\
\hline 2006 & $13(0.38)$ & $40(0.33)$ & $64(0.43)$ \\
\hline 2007 & $12(0.32)$ & $53(0.32)$ & $66(0.45)$ \\
\hline 2008 & $23(0.51)$ & $74(0.30)$ & $69(0.47)$ \\
\hline 2009 & $15(0.31)$ & $60(0.24)$ & $54(0.36)$ \\
\hline 2010 & $12(0.23)$ & $49(0.24)$ & $41(0.31)$ \\
\hline 2011 & $18(0.38)$ & $58(0.26)$ & $25(0.20)$ \\
\hline$P$ Value & 0.282 & 0.3727 & 0.02 \\
\hline \multicolumn{4}{|c|}{ Pelvic Pain (ICD-9 625.9, 789.00) } \\
\hline Total (2006-2011) & $3222(12.15)^{a b}$ & $13,891(11.52)^{\mathrm{ac}}$ & $11,750(13.81)^{\mathrm{bc}}$ \\
\hline 2006 & $384(11.12)$ & $1395(11.60)$ & $1987(13.35)$ \\
\hline 2007 & $466(12.25)$ & $1915(11.41)$ & $1997(13.52)$ \\
\hline 2008 & $519(11.60)$ & $2729(11.24)$ & $1976(13.47)$ \\
\hline 2009 & $627(12.88)$ & $2897(11.68)$ & $2117(14.23)$ \\
\hline 2010 & $661(12.60)$ & $2419(11.72)$ & $1881(14.13)$ \\
\hline 2011 & $565(12.07)$ & $2536(11.51)$ & $1792(14.27)$ \\
\hline$P$ Value & 0.1449 & 0.617 & 0.0593 \\
\hline \multicolumn{4}{|c|}{ Perforation of Uterine Wall (ICD-9 621.8, 665.3) } \\
\hline Total (2006-2011) & $412(1.55)^{\mathrm{ab}}$ & $1558(1.29)^{\mathrm{ac}}$ & $555(0.65)^{b c}$ \\
\hline 2006 & $40(1.16)$ & $142(1.18)$ & $57(0.38)$ \\
\hline 2007 & $51(1.34)$ & $176(1.05)$ & $76(0.51)$ \\
\hline 2008 & $48(1.07)$ & $301(1.24)$ & $91(0.62)$ \\
\hline
\end{tabular}


Table 2 Complications and side effects associated with IUD insertion and tubal sterilization over time (Continued)

\begin{tabular}{llll}
\hline & Copper IUD & LNG IUD & Tubal Sterilization \\
Complications/Side Effect & $\mathrm{n}(\%)$ & $\mathrm{n}(\%)$ & $\mathrm{n}(\%)$ \\
\hline 2009 & $88(1.81)$ & $319(1.29)$ & $119(0.80)$ \\
2010 & $98(1.87)$ & $307(1.49)$ & $103(0.77)$ \\
2011 & $87(1.86)$ & $313(1.42)$ & $109(0.87)$ \\
$P$ Value & 0.0014 & 0.0023 & $<0.0001$
\end{tabular}

${ }^{\mathrm{abc}} \mathrm{Chi}$ square pairwise comparisons between groups with the same superscript, $p<0.05$. The pairwise comparisons were done for the total for each complication/ side effect across years 2006-2011 and not for the individual years 